\title{
Definition of Neuronal Circuitry Controlling the Activity of Phrenic and Abdominal Motoneurons in the Ferret Using Recombinant Strains of Pseudorabies Virus
}

\author{
I. Billig, ${ }^{1}$ J. M. Foris, ${ }^{1}$ L. W. Enquist, ${ }^{4}$ J. P. Card, ${ }^{2,3}$ and B. J. Yates ${ }^{1,2}$ \\ Departments of ${ }^{1}$ Otolaryngology, ${ }^{2}$ Neuroscience, and ${ }^{3}$ Psychiatry, University of Pittsburgh, Pittsburgh, Pennsy/vania \\ 15213, and ${ }^{4}$ Department of Molecular Biology, Princeton University, Princeton, New Jersey 08544
}

\begin{abstract}
During a number of behaviors, including vomiting and some postural adjustments, activity of both the diaphragm and abdominal muscles increases. Previous transneuronal tracing studies using injection of pseudorabies virus (PRV) into either the diaphragm or rectus abdominis (RA) of the ferret demonstrated that motoneurons innervating these muscles receive inputs from neurons in circumscribed regions of the spinal cord and brainstem, some of which have an overlapping distribution in the magnocellular part of the medullary reticular formation (MRF). This observation raises two possibilities: that two populations of MRF neurons provide independent inputs to inspiratory and expiratory motoneurons or that single MRF neurons have collateralized projections to both groups of motoneurons. The present study sought to distinguish between these prospects. For this purpose, recombinant isogenic strains of $\mathrm{PRV}$ were injected into these respiratory muscles in nine ferrets; the strain injected into the
\end{abstract}

diaphragm expressed $\beta$-galactosidase, whereas that injected into RA expressed green fluorescent protein. Immunofluorescence localization of the unique reporters of each virus revealed three populations of infected premotor neurons, two of which expressed only one virus and a third group that contained both viruses. Dual-infected neurons were predominantly located in the magnocellular part of the MRF, but were absent from both the dorsal and ventral respiratory cell groups. These data suggest that coactivation of inspiratory and expiratory muscles during behaviors such as emesis and some postural adjustments can be elicited through collateralized projections from a single group of brainstem neurons located in the MRF.

Key words: pseudorabies virus; diaphragm; abdominal muscle; emesis; dorsal and ventral respiratory groups; medullary reticular formation; raphe nuclei; dual-labeling immunofluorescence
The diaphragm and abdominal muscles typically contract out of phase as they induce inspiration and expiration, respectively. These contractions are regulated by the brainstem dorsal and ventral respiratory groups, which generate the respiratory rhythm and also impose that rhythm on respiratory motoneurons (Feldman, 1986). However, the diaphragm and abdominal muscles also contract in phase during a number of behaviors, including emesis (Gold and Hatcher, 1926; McCarthy and Borison, 1974), some postural adjustments (Grillner et al., 1978), and in response to vestibular stimulation (Yates et al., 1993; Rossiter et al., 1996). Brainstem respiratory group neurons are not responsible for eliciting simultaneous increases in inspiratory and expiratory muscle activity during at least some of these responses. For example, bulbospinal inspiratory neurons in the dorsal and ventral respiratory groups are inhibited and mainly silent during emesis (Bianchi and Grèlot, 1989; Miller et al., 1990). Furthermore, vestibular stimulation can activate respiratory muscles without modulating the firing of respiratory group neurons (Yates et al., 1994; Woodring and Yates, 1997), and lesions of the main respiratory groups do not abolish vestibulo-respiratory reflexes (Yates et al., 1995; Rossiter et al., 1996; Shiba et al., 1996; Woodring and Yates, 1997).

Previous neuroanatomical studies in the rat employing the transneuronal transport of pseudorabies virus (PRV) injected into the diaphragm have revealed the locations of inspiratory neurons in the brainstem and spinal cord of this species (Dobbins and Feldman, 1994). It was reported that rat inspiratory premotor neurons were mainly located in the dorsal and ventral respiratory groups, al-

Received May 9, 2000; revised June 21, 2000; accepted July 21, 2000.

This work was supported by National Institutes of Health Grants R01 DC00693, R01 DC03732, and P01 DC03417 (B.J.Y.) and R01 NS33506 (L.W.E.). We thank Lucy Cotter and Kristine Hartge for excellent technical support during the course of these experiments.

Correspondence should be addressed to Dr. Bill Yates, University of Pittsburgh, Department of Otolaryngology, Room 106, Eye and Ear Institute, Pittsburgh, PA 15213. E-mail: byates@pitt.edu.

Copyright (C) 2000 Society for Neuroscience $0270-6474 / 00 / 207446-09 \$ 15.00 / 0$ though a few labeled neurons were present in the raphe nuclei, medial reticular formation, and parabrachial nucleus. Nevertheless, differences in the organization of neurons presynaptic to phrenic motoneurons were recently demonstrated in an emetic species, the ferret. In contrast to the rat, the ventral portion of the ferret medial medullary reticular formation (MRF), particularly the magnocellular division, contained a substantial number of infected neurons after the injection of PRV into the diaphragm, although both species exhibited infection in the region of the ventrolateral reticular formation known to contain the ventral respiratory group (Yates et al., 1999). In another group of experiments, neurons in the MRF were also infected by transynaptic passage of PRV from the ferret rectus abdominis ( $\mathrm{RA}$ ) muscle, along with neurons in portions of the ventrolateral reticular formation known to contain the ventral respiratory group, the nucleus retroambiguus, and the raphe nuclei (Billig et al., 1999). The overlapping distribution of labeled MRF neurons after injection of PRV into either the diaphragm or RA raises two possibilities: that single neurons in this area could have collateralized projections to both inspiratory and expiratory motoneurons or that two populations of neurons with overlapping distributions provide parallel inputs to inspiratory and expiratory motoneurons, as is the case for cells in the ventral respiratory group (Feldman, 1986).

In the present study, two antigenically distinct recombinant strains of PRV were used in dual injection paradigms to distinguish between these two prospects. This experimental approach is based on the ability of the two recombinant viruses to coinfect neurons with common synaptology (Fig. $1 A$ ). Recent studies have demonstrated the utility of this approach in mapping autonomic and visual circuitry (Jansen et al., 1995; Levatte et al., 1998; Ueyama et al., 1999) while also identifying the factors that may contribute to the generation of false negatives (Kim et al., 1999; Mabon et al., 1999). Although these data reveal the need to be conservative in interpreting negative findings, the demonstrated ability of two recombinants to coinfect neurons in these dual infection paradigms 


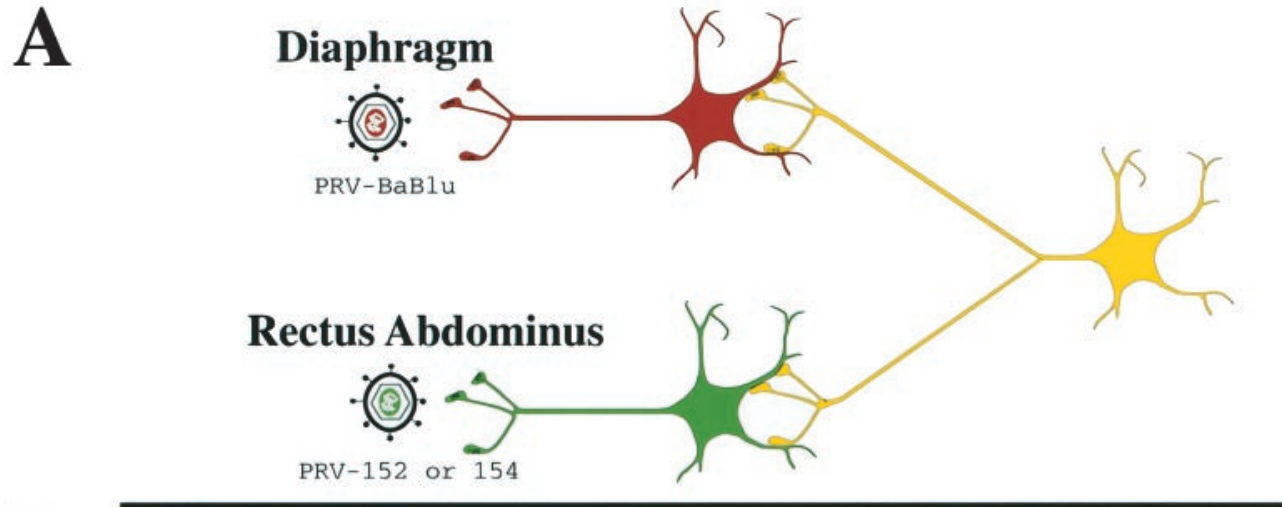

B
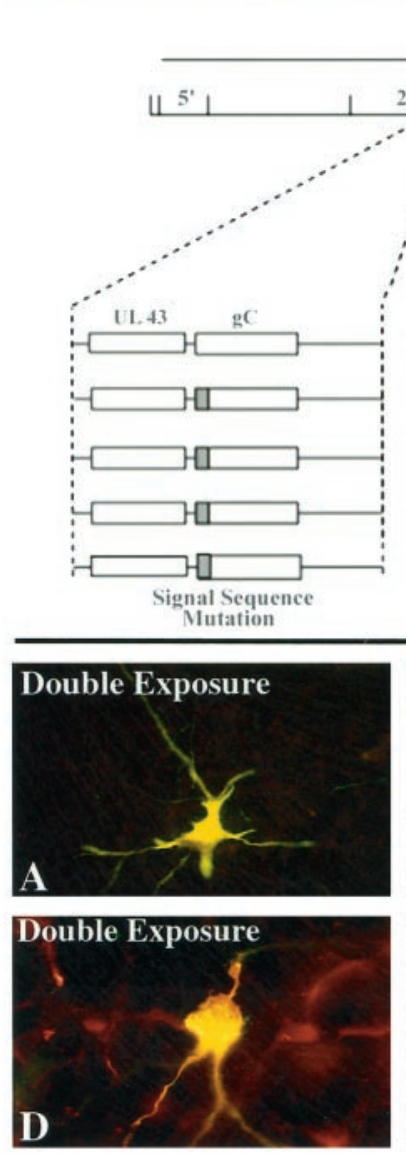
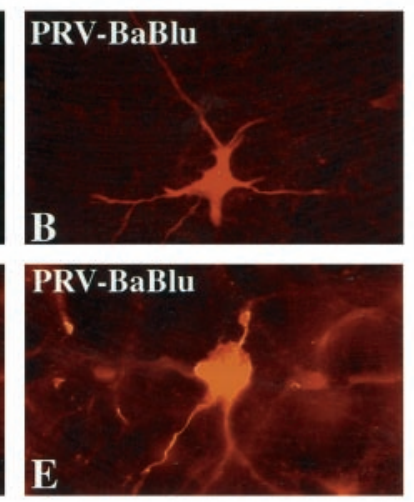
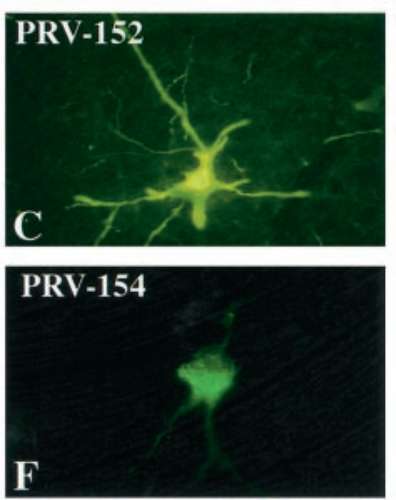

Figure 1. A, The experimental strategy used in this study. Two recombinant strains of pseudorabies virus were used to produce retrograde transynaptic infection of neurons synaptically linked to motoneurons innervating the diaphragm and rectus abdominis muscles. Each viral strain expressed a unique reporter that could be detected with the use of monospecific antisera. $B$, The genomic organization of the recombinant viruses used in this study. The genome of PRV contains unique long (UL) and unique short (US) regions. The parental strain used to produce the recombinants used in this study was an attenuated vaccine strain known as PRV-Bartha. PRV-Bartha has several well characterized mutations and deletions that distinguish it from the wild-type virus (PRV-Becker). These alterations are mapped on the diagram. All recombinants involved insertion of the transgene at the $\mathrm{gG}$ locus of the viral genome. In PRVBaBlu, $\mathrm{gG}$ was replaced with the gene encoding $\beta$-galactosidase. In PRV-152 and PRV-154, the gene encoding green fluorescent protein was inserted into the $\mathrm{gG}$ gene, either alone (PRV-152) or as part of a fusion protein (PRV-154). C, The distribution of the reporters within two infected neurons that were both double-labeled. $\beta$-galactosidase expression in neurons infected with PRV-Bablu provided staining of neuronal perikarya and dendrites (red fluorescence, panels $B$ and $E$ ). Two EGFP-expressing viruses (PRV-152 and PRV-154) labeled cells differently. PRV-152 produced staining of perikarya and dendrites comparable to that produced by PRV-Bablu (green fluorescence, $C)$. In contrast, the fusion protein produced by PRV-154 (EGFP+Us9) was differentially concentrated in the Golgi and rough endoplasmic reticulum of infected cells (green fluorescence, $F$ ). The majority of experiments reported here used PRV-152. Neurons containing both PRV-Bablu and PRV-152 or -154 appeared as having yellow fluorescence $(A$ and $D)$. provides a powerful means of addressing issues of collateralization that cannot be achieved with other anatomical methods. In the present study we have adapted this experimental approach to determine if a single population of brainstem neurons is presynaptic to two distinct populations of motoneurons involved in the control of respiration and emesis in the ferret.

\section{MATERIALS AND METHODS}

Animals. Experiments were conducted in 17 adult male ferrets (obtained from Marshall Farms, North Rose, NY). Animals were housed singly and allowed a minimum of 1 week acclimation to the animal facility before being injected with PRV. The experimental procedures used in this study conformed to regulations stipulated in the United States Department of Health and Human Services publication CDC 88-8395 and were approved by the University of Pittsburgh Institutional Animal Care and Use Committee.

Recombinant viruses. The experimental strategy is illustrated schematically in Figure $1 A$, and the organization of the genome of the recombinants used is shown in Figure $1 B$. Three recombinants of the Bartha strain of PRV (PRV-Bartha) that express either $\beta$-galactosidase $(\beta$-gal) or enhanced green fluorescent protein (EGFP) were used in this analysis. PRV-Bartha is an attenuated strain of PRV developed as a vaccine (Bar- tha, 1961). It has been used widely for transneuronal tracing (cf., Enquist et al., 1999; Card, 2000 for recent reviews), including in previous studies that provided the foundation for the present investigation (Billig et al., 1999; Yates et al., 1999). Preparation of PRV-BaBlu, a recombinant Bartha strain that expresses $\beta$-gal, has been described previously (Standish et al., 1995; Kim et al., 1999). Briefly, this strain contains the lacZ gene at the $\mathrm{gG}$ locus and produces $\beta$-gal under the control of the viral $\mathrm{gG}$ promoter. PRV-152 expresses EGFP. This virus carries an insertion at the $\mathrm{gG}$ locus such that EGFP is constitutively expressed using the cytomegalovirus immediate early promoter. The cell body, nucleus, and processes of cells infected with PRV-152 are filled with EGFP. PRV-154 expresses a novel membrane-anchored form of EGFP such that the trans-Golgi network is the only part of the infected cell that contains EGFP. This virus carries an insertion of a Us9-EGFP hybrid gene at the $\mathrm{gG}$ locus such that the fusion protein is expressed using the cytomegalovirus immediate early promoter (Brideau et al., 2000). PRV-Bartha, PRV-BaBlu, and PRV-152 do not express the Us9 gene; however, PRV-154 expresses two novel antigens, EGFP and Us9, that are localized predominately in the trans-Golgi network.

All viruses were grown in pig kidney (PK15) cells. The final titers in plaque-forming units (pfu) determined in PK15 cells were: PRV-BaBlu and PRV-152 = $1 \times 10^{8} \mathrm{pfu} / \mathrm{ml}, \mathrm{PRV}-154=3 \times 10^{8} \mathrm{pfu} / \mathrm{ml}$. Viruses were aliquoted at $100 \mu \mathrm{l} /$ tube and stored at $-80^{\circ} \mathrm{C}$. Individual aliquots of virus 


\begin{tabular}{|c|c|c|}
\hline Animal & Survival time (d) & Viruses injected $(\mu \mathrm{l})$ \\
\hline 1 & 4 & $\begin{array}{l}\text { PRV-Bablu: } 100 \\
\text { PRV-152: } 60\end{array}$ \\
\hline 2 & 4.5 & $\begin{array}{l}\text { PRV-Bablu: } 100 \\
\text { PRV-152: } 60\end{array}$ \\
\hline 3 & 5 & $\begin{array}{l}\text { PRV-Bablu: } 60 \\
\text { PRV-152: } 60\end{array}$ \\
\hline 4 & 5 & $\begin{array}{l}\text { PRV-Bablu: } 60 \\
\text { PRV-154: } 60\end{array}$ \\
\hline 5 & 5 & $\begin{array}{l}\text { PRV-Bablu: } 60 \\
\text { PRV-152: } 60\end{array}$ \\
\hline 6 & 5 & $\begin{array}{l}\text { PRV-Bablu: } 100 \\
\text { PRV-152: } 60\end{array}$ \\
\hline 7 & 5 & $\begin{array}{l}\text { PRV-Bablu: } 100 \\
\text { PRV-152: } 60\end{array}$ \\
\hline 8 & 5.5 & $\begin{array}{l}\text { PRV-Bablu: } 60 \\
\text { PRV-152: } 60\end{array}$ \\
\hline 9 & 5.5 & $\begin{array}{l}\text { PRV-Bablu: } 60 \\
\text { PRV-152: } 60\end{array}$ \\
\hline
\end{tabular}

were thawed immediately before injection. Excess virus was inactivated with Clorox and discarded.

Injection procedures. Initial experiments were conducted using eight animals in which a single virus, either PRV-Bablu or PRV-152, was injected into the left RA to determine if the two recombinants were transported at the same rate. Animals were then killed after survival times of $3(n=1), 4(n=1), 5(n=4)$, or $6 \mathrm{~d}(n=2)$. Subsequently, nine additional experiments were conducted in which PRV-Bablu was injected into the diaphragm, and PRV-152 or PRV-154 was injected into the RA muscle of the same animal. These animals were killed $4(n=1), 4.5(n=$ $1), 5(n=5)$, or $5.5(n=2)$ d after inoculation. Further detail regarding the strain and volume of viruses injected in these experiments is provided in Table 1.

The injection procedures conformed to those detailed in our previous viral transneuronal analyses of premotor circuits that modulate the activity of diaphragm and RA motoneurons (Billig et al., 1999; Yates et al., 1999) Animals were initially anesthetized using a mixture of ketamine (25 $\mathrm{mg} / \mathrm{kg}$ ) and xylazine $(2.5 \mathrm{mg} / \mathrm{kg})$ injected intramuscularly; anesthesia was supplemented with $0.5-1 \%$ isoflurane, which was vaporized in $\mathrm{O}_{2}$ and administered through a face mask to maintain areflexia. In cases in which the diaphragm was injected with PRV, a midline incision was made through the linea alba, and the ventral surface of the left diaphragm was exposed by retracting the viscera. PRV-Bablu was injected beneath the peritoneal lining of the diaphragm using a $10 \mu \mathrm{l}$ Hamilton syringe equipped with a 26 gauge needle. Injections of virus (1-2 $\mu \mathrm{l} /$ injection) were made at multiple sites in both the costal and crural regions of the diaphragm ipsilaterally. The total volume of virus injected was $60-100 \mu \mathrm{l}$. Similar procedures were used to inject $60 \mu \mathrm{l}$ of PRV-152 or PRV-154 into the left RA; these injections were made beneath the connective tissue sheath surrounding the muscle and were restricted to the region within 3 $\mathrm{cm}$ of the diaphragm. After the injections, the abdominal musculature and skin were closed using sutures, and animals were maintained under Biosafety level II conditions for the balance of the survival period.

Tissue preparation. After their respective survival times, animals were deeply anesthetized using ketamine $(35 \mathrm{mg} / \mathrm{kg})$ and xylazine $(5 \mathrm{mg} / \mathrm{kg})$ injected intramuscularly, and then perfused transcardially with saline followed by paraformaldehyde-lysine-periodate (PLP) fixative (McLean and Nakane, 1974), as previously described (Billig et al., 1999; Yates et al., 1999). The brainstem and cervical, thoracic, and lumbar cord segments were removed, post-fixed $4-5 \mathrm{hr}$ or overnight at $4^{\circ} \mathrm{C}$ in PLP, and cryoprotected by immersion in a $30 \%$ sucrose-PBS solution at $4^{\circ} \mathrm{C}$ for $2 \mathrm{~d}$. Transverse $50 \mu \mathrm{m}$ sections of the spinal cord and brainstem were cut with a freezing microtome, collected sequentially in four wells of cryopreservant (Watson et al., 1986), and stored at $-20^{\circ} \mathrm{C}$ until processed for immunohistochemical localization of viral antigens or reporter proteins.

Immunohistochemistry: single virus injection experiments. In experiments in which a single viral strain was injected (into RA), neurons infected with PRV-Bablu or PRV-152 were visualized using immunoperoxidase procedures described in detail elsewhere (Enquist and Card, 1996; Card and Enquist, 1999). Briefly, one well of sections was incubated in rabbit polyclonal anti-pseudorabies virus antiserum $(\mathrm{Rb}-133)$ diluted to a final concentration of $1: 10,000$. Thereafter, sections were processed using the avidin-biotin modification of the peroxidase anti-peroxidase procedure
(Hsu et al., 1981), which used affinity-purified biotinylated donkey antirabbit IgG (Jackson ImmunoResearch, West Grove, PA) and Vectastain reagents (Elite kit; Vector Laboratories, Burlingame, CA) for immunoperoxidase localization of viral antigen. On completion of the immunohistochemical processing, the tissue was mounted on gelatin-coated slides, dehydrated, cleared, and coverslipped using Cytoseal 60 (VWR Scientific, West Chester, PA).

Immunohistochemistry: double virus injection experiments. In experiments in which different recombinants were injected into the diaphragm and RA, dual-labeling immunofluorescence techniques were used to visualize infected neurons. One bin of free-floating sections (section frequency of $200 \mu \mathrm{m}$ ) was incubated for $2 \mathrm{~d}$ at $4^{\circ} \mathrm{C}$ in a combination of mouse anti- $\beta$-galactosidase (Sigma, St. Louis, MO; 1:1,500) and rabbit anti-green fluorescent protein (Clontech, Palo Alto, CA; 1:1000 or Molecular Probes, Eugene, OR; 1:250) to localize PRV-Bablu or either PRV-152 or PRV-154, respectively. Sections were then washed thoroughly in PBS before being incubated in affinity-purified secondary antibodies raised in donkey that were conjugated to either the CY3 (red) or CY2 (green) carbocyanine (Jackson ImmunoResearch). As a convention in this study, secondary antibodies conjugated to CY3 (concentration of 1:500) were used to visualize PRV-BaBlu, and secondary antibodies conjugated to CY2 (concentration of 1:300) were used to visualize PRV-152 and -154. The incubations in these species-specific secondary antibodies were conducted simultaneously for $2 \mathrm{hr}$ at room temperature before the sections were washed and mounted on gelatin-coated slides, and then dehydrated, cleared, and coverslipped with Cytoseal 60. An adjacent bin of brainstem tissue was stained for Nissl substance and fiber tracts using a modified Kluver-Barrera procedure (Kiernan, 1990), so that the precise boundaries of neuronal structures could be determined.

Several controls were included in the analysis to establish the efficiency of transgene expression and the subcellular localization of the reporter proteins. These included: (1) dual labeling immunofluorescence localization of viral antigens with either $\beta$-galactosidase or EGFP to demonstrate that the transgenes were efficiently expressed in all infected neurons, (2) immunoperoxidase localization of $\beta$-galactosidase or EGFP in sections adjacent to those processed for immunofluorescence, (3) immunoperoxidase localization of infected neurons in adjacent sections of brainstem with a rabbit polyclonal antiserum (Rb133) raised against acetone-inactivated PRV, and (4) localization in PRV-154 infected neurons of EGFP to the Golgi apparatus and not to the processes or nucleus. Collectively, these localizations demonstrated that the distribution of infected neurons pro-

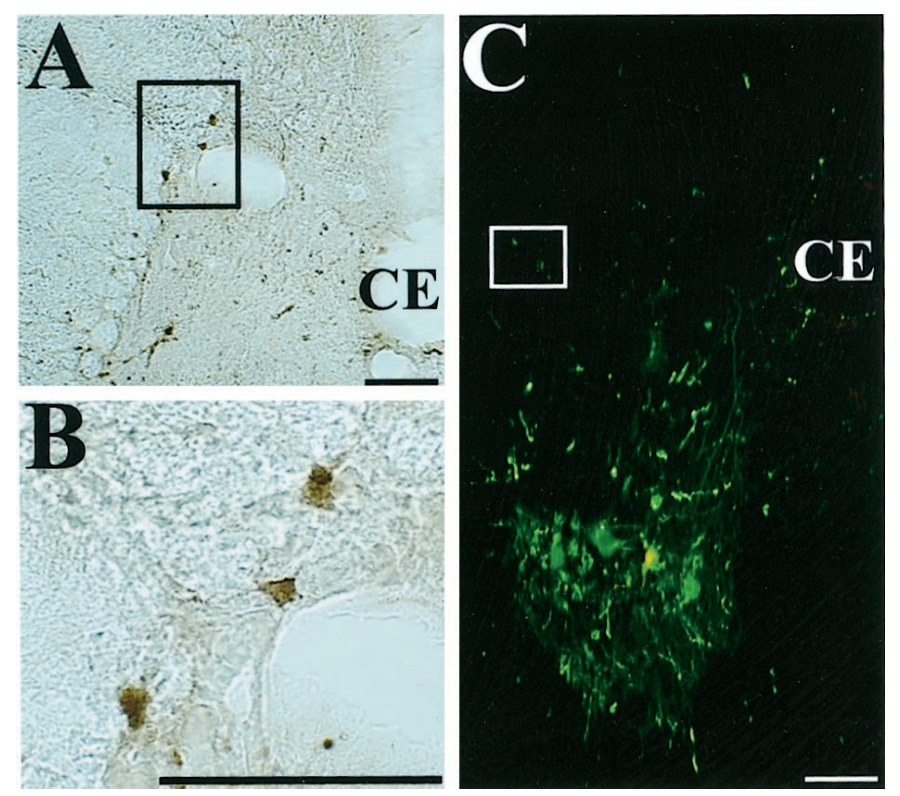

Figure 2. Photomicrographs illustrating restricted infection of sympathetic preganglionic neurons after injection of PRV into respiratory muscles. $A, B$, Infected neurons in the intermediolateral cell column of the $T_{8}$ spinal segment after injection of PRV-152 into rectus abdominis; the section was immunoprocessed using immunoperoxidase. $B$ is a higher magnification view of the region in $A$ indicated by a box. Note that the infection was confined to the nucleus, cell body, and proximal dendrites of the labeled sympathetic preganglionic neurons. $C$, Infected neurons in the $T_{11}$ spinal cord segment after the combined injection of PRV-Bablu into the diaphragm and PRV-152 into rectus abdominis. The section was immunoprocessed using immunofluorescence and photographed under illumination that excited both the CY2 and CY3 fluorophors. Note that the labeling of sympathetic preganglionic neurons in the intermediolateral cell column, which is indicated by a box, is much weaker than that of motoneurons located in the ventral horn. Scale bars, $100 \mu \mathrm{m}$. 

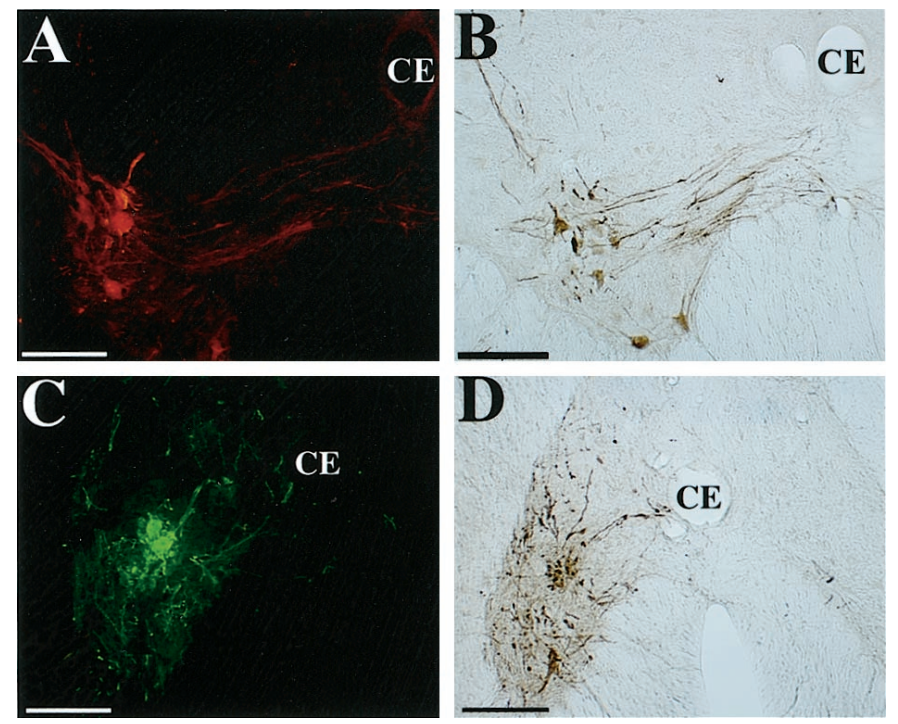

Figure 3. Photomicrographs of presumed motoneurons infected 4 and $4.5 \mathrm{~d}$ after injection of PRV-Bablu into the diaphragm and PRV-152 into rectus abdominis. $A, B$, Large presumed motoneurons immunostained with the red CY3 fluorophor $(A)$ or immunoprocessed with peroxidase $(B)$ after an injection of PRV-Bablu into the diaphragm. The presumed phrenic motoneurons were located in the ventromedial ventral horn of the $\mathrm{C}_{6}$ spinal cord segment ipsilateral to the side of the injection. These large neurons characteristically exhibited fasciculated bundles of dendrites that extended toward the central canal $(C E)$ and sometimes crossed the midline to the contralateral side. $C, D$, Examples of a cluster of presumed motoneurons, immunostained with the green CY2 fluorophor $(C)$ or immunoprocessed with peroxidase $(D)$ after an injection of PRV-152 into the rectus abdominis. The cells were observed in ipsilateral lamina V III of the $\mathrm{T}_{10}$ spinal cord segment. These large neurons exhibited large dendritic arbors, with some processes crossing the midline or reaching the central canal $(C E)$. Scale bars, $200 \mu \mathrm{m}$.

duced by each recombinant recapitulated the projection-specific distribution of neurons revealed in our previous studies (Billig et al., 1999; Yates et al., 1999). Importantly, these data also demonstrated that the distribution of neurons expressing transgenes was coextensive with the distribution of infected neurons revealed by the rabbit polyclonal antiserum. Thus, these data demonstrate that transgene expression provides an accurate measure of the full extent of viral replication and transynaptic passage.

The subcellular localization of reporter proteins differed among the recombinants. The $\beta$-gal and EGFP expressed by PRV-BaBlu and PRV152 , respectively, produced extensive staining of the somata (cell nucleus and cytoplasm) and dendrites of infected neurons (Fig. $1 C$, panels $B, C, E$ ). In contrast, the Us9-EGFP fusion protein produced by PRV-154 was differentially concentrated within the Golgi complex and perinuclear membranes of infected neurons; the nucleus was not labeled (Fig. $1 C$, panel $F$ ). The differential localization of the Us9-EGFP fusion protein is consistent with the demonstration by Brideau et al. (1998) that Us9 localizes to the trans-Golgi network and endocytotic pathways in PRV-infected neurons, but not to the endoplasmic reticulum or nuclear membranes. The extensive cellular staining produced by PRV-152 had the added advantage of providing detail on the morphology of infected neurons. For this reason, we used PRV-152 in the majority of the dual infection studies. However, the cell body and non-nuclear staining exhibited by PRV-154 was useful in identifying neurons that were co-infected with PRV-BaBlu, and thus this virus was used in a limited number of cases.

Tissue analysis. The primary analysis was done on sections that were spaced $200 \mu \mathrm{m}$ apart, both in animals injected with a single virus or two viruses. Previous studies in the ferret have shown that this frequency is sufficient for an accurate localization of all cell groups synaptically linked to motoneurons innervating the diaphragm (Yates et al., 1999) or the RA muscle (Billig et al., 1999). Two additional series of thoracic spinal cord tissue from all double virus-injected animals were processed using immunoperoxidase procedures to determine whether labeling was present in sympathetic preganglionic neurons located in the intermediolateral column (IML), and an additional group of brainstem sections was processed to determine the extent of infection of parasympathetic pathways. In addition to addressing the potential contribution of autonomic pathways to brainstem infection, this analysis provided a more comprehensive analysis of the distribution of brainstem circuitry involved in the transynaptic infection.

Sections were examined and photographed using a Zeiss Axioplan photomicroscope equipped with epifluorescence and differential interference contrast (DIC) optics. Immunoperoxidase preparations were photographed using transmitted light and fluorescence material was photographed using epifluorescence in combination with filters that selectively
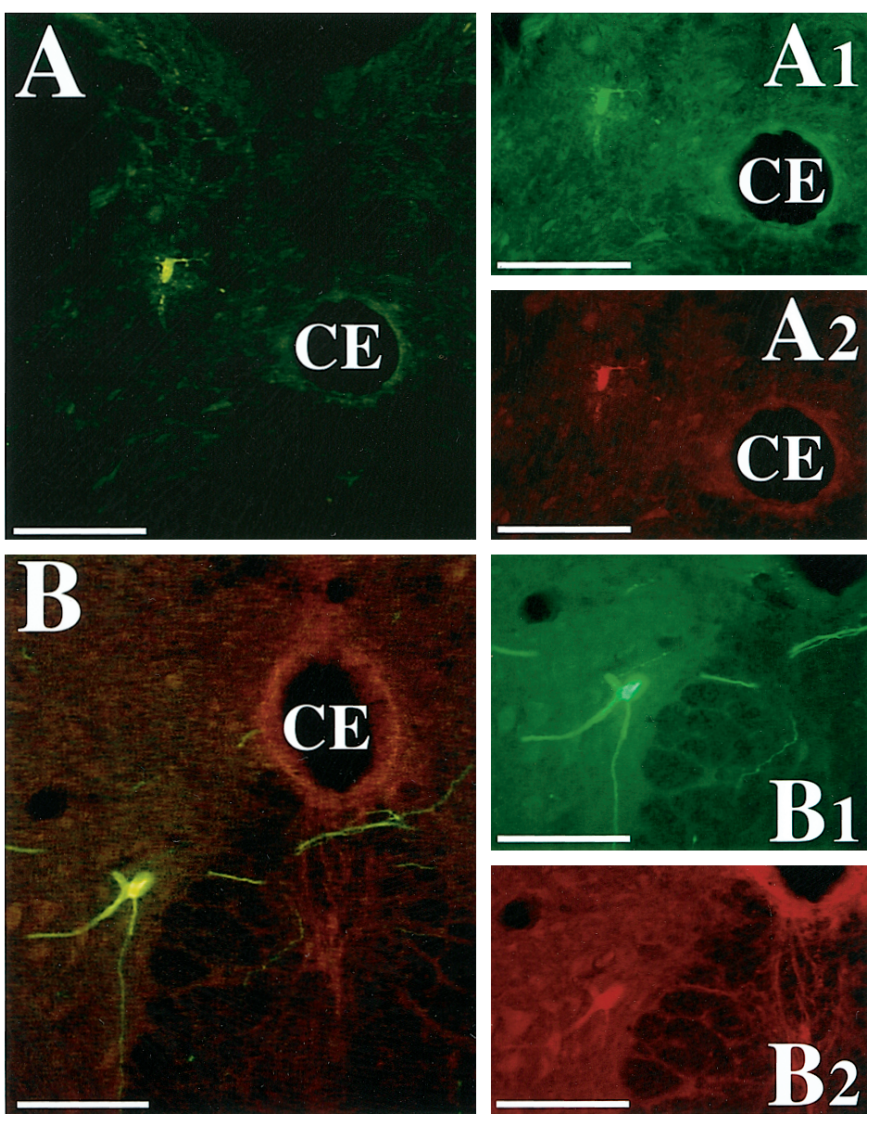

Figure 4. Photomicrographs of presumed spinal cord interneurons infected 4.5 and $5 \mathrm{~d}$ after PRV-Bablu injection into the diaphragm and PRV-152 injection into rectus abdominis. These neurons were dually immunostained with the red CY3 and the green CY2 fluorochromes, indicating that they made synaptic connections with both phrenic and abdominal motoneurons. The cells were located in lamina VII of the $\mathrm{T}_{8}(A)$ and lamina VIII of the $\mathrm{L}_{2}(B)$ spinal cord segments, ipsilateral to the injections. $A 1-B 2$ show the cell under illumination that excites one of the two fluorophors, whereas $A$ and $B$ were photographed under illumination that excites both fluorophors. Scale bars, $200 \mu \mathrm{m}$.

excited CY2 or CY3. In the dual-labeling immunofluorescence analysis, each field was photographed in single exposures that recorded the location of cells harboring each reporter protein and in double exposures that revealed the cellular localization of both viruses (Fig. 1C). Verification that yellow fluorescence reflected the colocalization of both the CY2 and CY3 fluorophors and was not attributable to the presence of overlapping cells that each contained one of the fluorophors, was established using a $40 \times$ objective. Images were also digitized using a Dage MTI 3CCD camera (Mutech, Billerica, MA) and a Simple32 image analysis system (Compix, Lake Oswego, OR). Images were prepared for publication using Adobe Photoshop software. Individual images were adjusted for size and contrast, but color balance was not altered.

In the dual virus injection experiments, only a qualitative analysis of the locations of neurons expressing immunoreactivity to one or both viruses was attempted. As noted in the introductory remarks, infection of a neuron by one strain of PRV can lower its susceptibility to infection by a second strain (Kim et al., 1999). Thus, it is possible that some neurons with divergent projections to both diaphragm and RA motoneurons may not have been double-labeled in these experiments. The prospect of false negatives mandates a conservative interpretation of the data but does not detract from the significance of dual infected neurons. However, the presence of false negatives could produce misleading results regarding the relative proportion of a cell group that contains neurons that collateralize to innervate both populations of motoneurons. Therefore, we have refrained from making quantitative determinations of the number of dualinfected neurons. Nevertheless, it should be emphasized that the patterns of infection described and illustrated in this report are representative of all animals included in the analysis.

\section{RESULTS}

\section{Involvement of autonomic pathways}

A thorough analysis of both the thoracic spinal cord and brainstem revealed only minor infection within autonomic pathways after 
Figure 5. Examples of premotor neurons in regions of the brainstem known to contain the dorsal and ventral respiratory groups, which were infected $4.5 \mathrm{~d}$ after PRV injections into the diaphragm and rectus abdominis. $A-D$ show photomicrographs of transverse brainstem sections located $\sim 1 \mathrm{~mm}$ caudal to the obex $(A, B)$ or at the level of the obex $(C, D)$; these sections were stained with the use of a modified Kluver-Barrera method. Boxes on the photomicrographs indicate the locations of the infected neurons illustrated in the middle column; these sections were immunoprocessed using immunofluorescence. The right column illustrates infected neurons at the same location in sections immunoprocessed using immunoperoxidase. $A 1$ and $A 2$ show neurons in nucleus retroambiguus that were infected after injecting PRV-152 into rectus abdominis on the contralateral side. The neurons were immunostained with the green CY2 fluorophor $(A 1)$ or with peroxidase $(A 2) . B 1$ and $B 2$ show premotor neurons in nucleus retroambiguus infected after injecting PRV-Bablu into the diaphragm on the contralateral side; these neurons were labeled with the red CY3 fluorophor (B1) or through immunoperoxidase processing (B2). $C 1$ and $C 2$ illustrate infected premotor neurons in the vicinity of nucleus ambiguus and the retrofacial nucleus that were immunostained with the red CY3 fluorophor $(C 1)$ or with peroxidase (C2) after injections of PRV-Bablu into the contralateral diaphragm. D1 and D2 show infected neurons in the ventrolateral portion of the nucleus of the solitary tract, which were immunostained with the red CY3 fluorophor $(D 1)$ or with peroxidase (D2) after injecting PRV-Bablu into the ipsilateral diaphragm. Scale bars, $400 \mu \mathrm{m}$. 12 , Hypoglossal nucleus; $5 S L$, laminar spinal trigeminal nucleus; $5 S P$, alaminar spinal trigeminal nucleus; $A$, nucleus ambiguus; $A P$, area postrema; $C E$, central
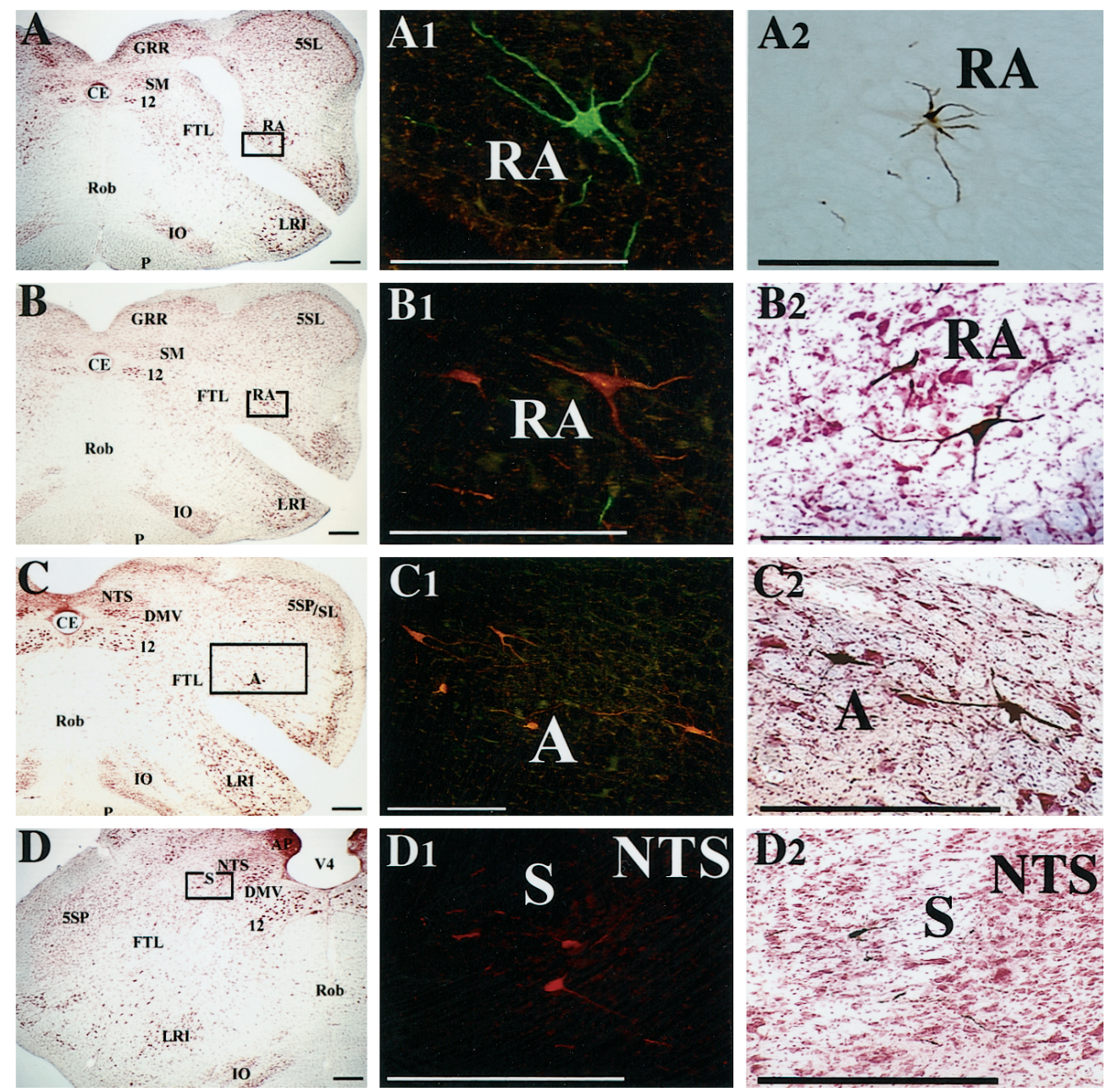
$L R I$, internal division of lateral reticular nucleus; $N T S$, nucleus of the solitary tract; $P$, pyramid; $R A$, nucleus retroambiguus; Rob, nucleus raphe obscurus; $S$, solitary tract; $S M$, medial nucleus of solitary tract; $V 4$, fourth ventricle.

injection of PRV into the diaphragm and RA. No infected parasympathetic preganglionic neurons were observed in the brainstem of any of the animals. Varying degrees of infection were observed in the IML of the thoracic spinal cord. Infected sympathetic preganglionic neurons were observed in all experimental animals, but the number of cells was small and the intracellular distribution of viral antigens detected with the rabbit polyclonal anti-PRV antiserum was characteristic of early stages of infection. Specifically, only scattered labeled neurons were observed in a subset of sections through the IML and, in those cases, viral immunoreactivity was largely restricted to the nucleus, cytoplasm, and proximal dendrites of the infected neurons, as illustrated in Figure 2. This restricted distribution of viral antigens in these neurons was in marked contrast to the extensive distribution observed within infected motoneurons from the same animals (Fig. 3). Even at the earliest survival intervals examined, infected motoneurons characteristically exhibited dense staining throughout the somatodendritic compartment, including distal branches of the dendritic arbor. Because the intracellular distribution of viral antigens is a reflection of the timing of infection (i.e., early stages of infection are associated with a restricted distribution of immunoreactivity) (Card, 1995; Card and Enquist, 1999), these data indicate that the replication and transynaptic transport of virus through motor circuits was more advanced than that in autonomic circuitry. Further evidence that brainstem labeling was not attributable to transneuronal passage of virus from sympathetic preganglionic neurons lies in the distribution of infected neurons, which differed substantially from that documented in viral transynaptic analysis of autonomic circuitry (Loewy, 1990; Jansen et al., 1995).

\section{Patterns of neuronal infection}

The distribution of infected neurons revealed by injection of the recombinant viruses into the diaphragm and RA duplicated the projection-specific patterns of transport that resulted from individual injection of the parental virus into these muscles (Billig et al., 1999; Yates et al., 1999). This occurred irrespective of whether the recombinants were injected individually or in dual injection paradigms. After injection of virus into the diaphragm, infected large presumed motoneurons were present in the ventral horn of segments $\mathrm{C}_{5}$ through $\mathrm{C}_{7}$ (but mainly $\mathrm{C}_{6}$ ) ipsilateral to the injection, whereas injection of virus into the proximal $3 \mathrm{~cm}$ of RA-infected neurons concentrated largely in the ventral horn of the $T_{10}$ to $T_{14}$ segments on the ipsilateral side. Examples of infected motoneurons are illustrated in Figure 3. The morphology and disposition of these neurons within the ventral horn also differed depending on the injected muscle. Infected diaphragm motoneurons were confined to a tight column in the ventromedial portion of the ventral gray and gave rise to fasiculated bundles of dendrites that exhibited a polarized trajectory toward the area immediately subjacent to the central canal. In contrast, infected RA motoneurons were more widely dispersed throughout the central and lateral portions of the ventral gray and gave rise to dendrites that extended radially from the somata. In both cases, the neuronal perikarya were 40-60 $\mu \mathrm{m}$ in widest diameter and exhibited morphological features classically defined for motoneurons using classical retrograde tracers. The number of infected cells exhibiting this morphology increased with advancing survival, but the segmental distribution and disposition 

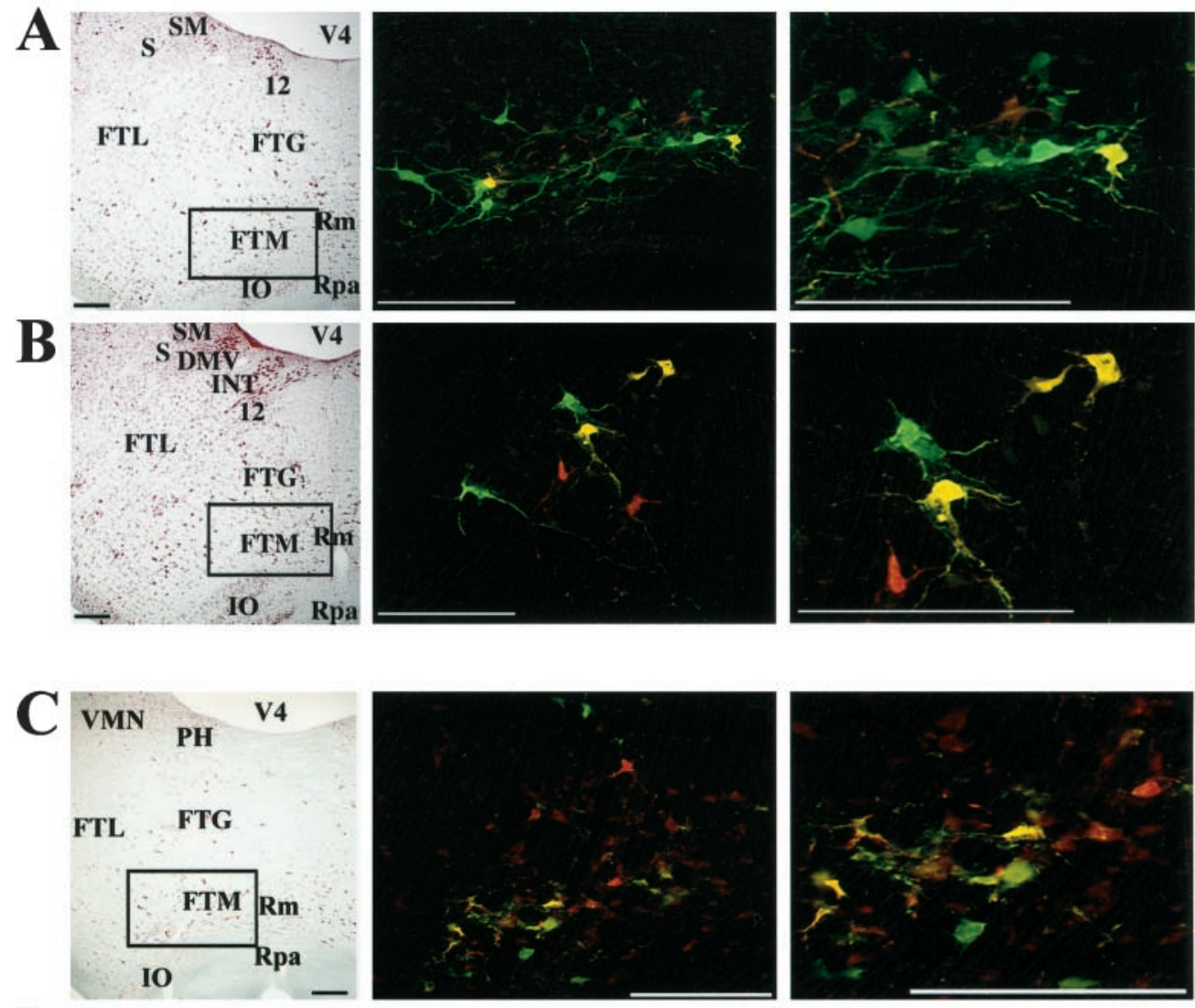

Figure 6. Location of premotor neurons in the MRF infected after injection of PRV into the diaphragm and rectus abdominis. $A$ and $B$ show infected neurons after a $4.5 \mathrm{~d}$ survival time, whereas $C-E$ illustrate infection after a $5 \mathrm{~d}$ survival. In addition, $A$ and $D$ show labeling at $\sim 2.5$ mm rostral to the obex, whereas $B$ and $E$ show labeling at $\sim 2.0 \mathrm{~mm}$ rostral to the obex, and $C$ illustrates labeling at $\sim 3.0$ $\mathrm{mm}$ rostral to the obex. Left column, Photomicrographs of brainstem sections (on the side ipsilateral to the injections) stained with a modified Kluver-Barrera method. Boxes surround the area containing infected neurons illustrated in the photomicrographs immediately on the right. Middle and right columns, Photomicrographs of infected neurons observed in the MRF. Red cells were only infected by retrograde transynaptic passage of PRVBablu from the diaphragm, green cells were only infected by retrograde transynaptic passage of PRV-152 from rectus abdominis, and yellow cells contain both viruses. Photomicrographs in the right column are a magnification of those in the middle column. Note that after a $5 \mathrm{~d}$ survival time, labeling is more prevalent and extends further rostrally than after a $4.5 \mathrm{~d}$ survival. Scale bars, $400 \mu \mathrm{m}$. Abbreviations are the same as in Figure 4, with the following additions: $F T G$, gigantocellular tegmental field; FTM, magnocellular tegmental field; INT, nucleus intercalatus; $R m$, nucleus raphe magnus; $R p a$, nucleus raphe pallidus.

of the cells in the ventral horn remained projection-specific throughout the longest postinoculation intervals.

At postinoculation intervals extending to $5.5 \mathrm{~d}$, transynaptic passage and replication of the recombinant viruses led to the appearance of infected neurons in the spinal cord and brainstem. The distribution of these neurons correlated with that demonstrated in our previous reports of this circuitry derived from injections of the parental virus into the diaphragm or RA (Billig et al., 1999; Yates et al., 1999). The infected neurons included local circuit neurons at multiple levels of the spinal cord (Fig. 4) as well as reproducible groups of neurons in the caudal brainstem (Figs. $5,6)$. The brainstem labeling was first apparent $4 \mathrm{~d}$ after injection of either muscle, and the number of infected neurons increased progressively through $5.5 \mathrm{~d}$. Neurons were observed bilaterally with an ipsilateral propensity. In both paradigms, some infected neurons were observed in brainstem regions known to contain the dorsal and ventral respiratory groups in a variety of mammalian species (Feldman, 1986), and substantial numbers of neurons were observed in the MRF and along the midline in the vicinity of the raphe obscurus and raphe pallidus.

\section{Dual infection experiments}

Immunofluorescence analysis with filters selective for the CY2 and CY3 fluorophors and with a wide-band Omega filter that excites both fluorophors allowed a detailed analysis of neurons infected with one or both of the recombinant viruses. As noted in Materials and Methods, the CY2 fluorophor was used to identify neurons infected with either PRV-152 or PRV-154, whereas CY3 was used to localize the $\beta$-galactosidase reporter expressed by PRV-BaBlu. Additionally, PRV-BaBlu injection was restricted to the diaphragm, and the EGFP-expressing recombinants were only injected into RA. Thus, neurons that were a dedicated part of 


\section{5 days}

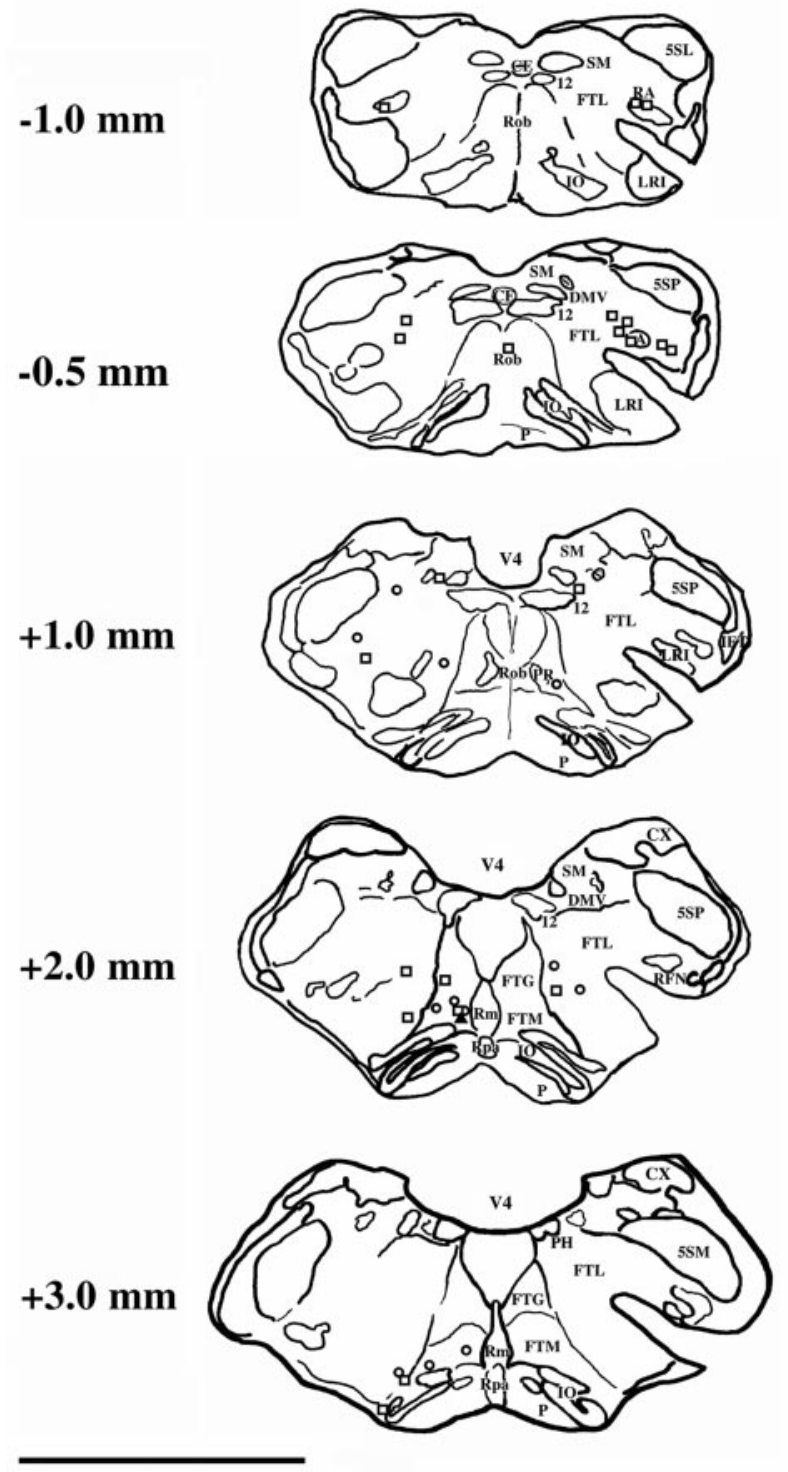

\section{0 days}
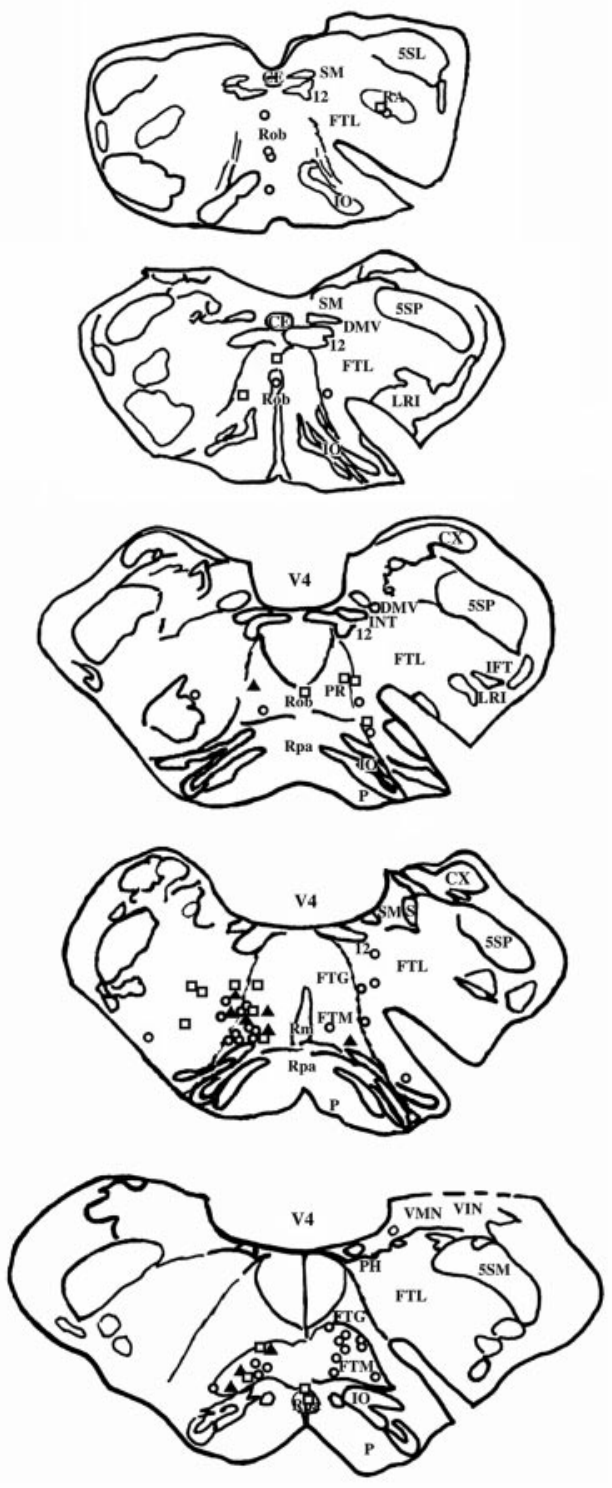

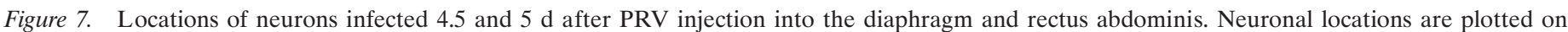

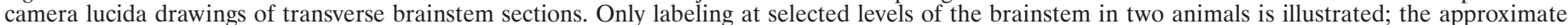

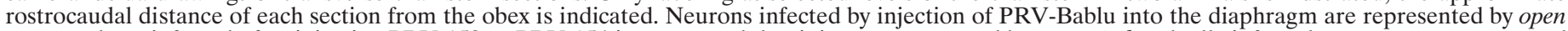

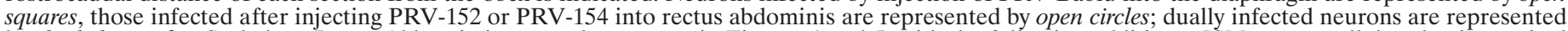

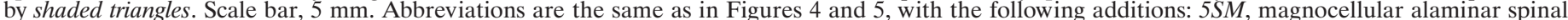

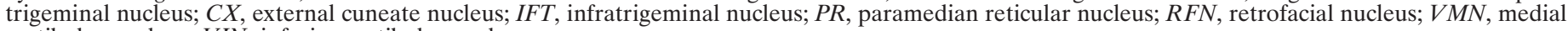
vestibular nucleus; VIN, inferior vestibular nucleus.

polysynaptic circuits selectively innervating the diaphragm appeared red, those infected selectively by transynaptic passage of virus from RA appeared green, and neurons that collateralized to innervate motoneurons to both muscles appeared yellow. Each of the dual-injected animals that survived $\geq 4.5 \mathrm{~d}$ contained neurons that were replicating both recombinants. Furthermore, sections that contained dual-infected neurons always also contained neurons selectively labeled with $\beta$-galactosidase or EGFP. Consequently, we are confident that both recombinants had replicated and passed transynaptically into comparable levels of the brainstem in all experimental animals. Nevertheless, we cannot preclude the possibility that previous infection of some neurons by one strain of virus made those cells refractory to replication of the second strain, as was recently demonstrated by Kim et al. (1999). However, it is noteworthy that the interference in viral replication demonstrated by Kim et al. (1999) was observed in animals in which one strain of virus was more virulent and transported at a faster rate than the other strain. In this study, both recombinants were produced from the same attenuated virus (PRV-Bartha) and exhibited the same relative rate of transport.

As noted above, some infected neurons were observed in brainstem regions known to contain the dorsal and ventral respiratory groups. Further immunofluorescence analysis revealed that these cells consisted of two projection-specific populations, and no cells contained reporters for both recombinants. Neurons selectively expressing either $\beta$-galactosidase or EGFP immunoreactivity were observed in the vicinity of the ventral respiratory group, whereas most neurons in the vicinity of the dorsal respiratory group ex- 
pressed $\beta$-galactosidase immunoreactivity (Fig. 5). This observation contrasted with the labeling observed in the more rostrally situated sections through the MRF of the same animals. In each animal, three populations of neurons were observed in the magnocellular tegmental field in the MRF: those replicating only one recombinant and those that contained the reporter proteins of both recombinants (i.e., dual-infected neurons were intermixed with cells that only expressed the EGFP or $\beta$-galactosidase reporters), as illustrated in Figure 6. However, it was uncommon to observe dual-labeled neurons along the midline in the vicinity of the raphe cell groups, although neurons expressing immunoreactivity to one of the two reporters were present in this area. Figure 7 shows the distribution of infected neurons at selected levels of the brainstem in two animals.

\section{DISCUSSION}

This study provides the first direct evidence that a population of neurons in the brainstem, located mainly in the magnocellular part of the MRF, provides inputs to both inspiratory and expiratory motoneurons in the spinal cord. Furthermore, the patterns of dual infection of brainstem neurons reported here support the conclusion that cells synaptically linked to both the diaphragm and RA exhibit a functional segregation from neurons that provide inputs to only one of these muscles. This functional parcellation is characterized by segregation of circuitry responsible for the generation of the respiratory rhythm from that responsible for coordinating cocontraction of multiple respiratory muscles during behaviors such as emesis.

Two caveats must be considered when interpreting the results of this study. First, after the injection of the recombinant viruses into the diaphragm and rectus abdominis the infection of neurons in the dorsal and ventral respiratory groups was not as extensive as when the parental stain, PRV-Bartha, was used (Billig et al., 1999; Yates et al., 1999). It is possible that the recombinant viruses were not transported as rapidly as the parental strain and that a longer transport time would have resulted in a more extensive infection of the respiratory groups. Nonetheless, the distribution of labeling produced by injection of PRV-Bartha and the recombinants PRV152, PRV-154, and PRV-Bablu into respiratory muscles was similar, and there is no indication that the general results would have been different from those in the present experiments had longer survival times been used. A second caveat is that a limited amount of infection of sympathetic nervous system neurons did occur in these studies, raising the prospect that some of the labeling of brainstem neurons observed in this study was attributable to transneuronal passage of virus from sympathetic preganglionic neurons in the thoracic spinal cord. Although we cannot eliminate this possibility, two lines of evidence raised in Results support our conclusion that the MRF neurons were infected predominantly by virtue of their collateralized projections to motoneurons controlling the diaphragm and rectus abdominis muscles.

Although previous experiments using PRV injections have shown that overlapping populations of MRF neurons influence RA and diaphragm activity (Billig et al., 1999; Yates et al., 1999), these conventional transneuronal tracing studies using one virus could not distinguish whether parallel projections from two groups of neurons or collateralized projections from a single group provided inputs to inspiratory and expiratory motoneurons. Furthermore, the present findings have physiological importance, because they shed light on lesion studies showing that inactivation of the MRF abolishes simultaneous increases in diaphragm and abdominal muscle activity during emesis (Miller et al., 1996). The current data demonstrate that individual MRF neurons may simultaneously activate inspiratory and expiratory motoneurons during vomiting, opening the possibility that pharmacological agents acting on this single neuronal population could abolish this behavior. Nevertheless, additional electrophysiological and lesion studies will be required to determine the role of MRF neurons in coordinating the contractions of the diaphragm and abdominal muscles during vomiting (Gold and Hatcher, 1926; McCarthy and Borison, 1974), as well as during postural adjustments (Grillner et al., 1978), and reaction to vestibular stimulation (Yates et al., 1993; Rossiter et al., 1996). In addition, it remains to be determined whether abdominal muscles other than RA receive substantial inputs from MRF premotor neurons, including those that also influence diaphragm activity.

It seems likely that the methodology used in this study can be used to determine whether any two muscles or muscle groups receive common influences from particular CNS regions. However, several limitations are apparent in this approach. Because infection of a neuron by one virus can lower its susceptibility to be infected by a second virus (Kim et al., 1999), it is only possible to interpret positive results (i.e., the presence of double-labeled neurons) when using this method. Furthermore, because the immune system of the ferret rapidly and effectively compartmentalizes and eliminates neurons that are infected with PRV (Billig et al., 1999; Yates et al., 1999), the temporal window during which infected neurons can be detected is limited. As a result, experiments incorporating injection of two virus tracers cannot be used in isolation to determine neural pathways that are involved with coordinating movements. Nonetheless, these experiments may provide clues regarding potential neural pathways that can be investigated in subsequent lesion, electrophysiological, and conventional neuroanatomical studies.

In conclusion, the present demonstration of dual infections of MRF neurons by antigenically distinct recombinants injected into the diaphragm and RA provides novel insights into the functional organization of brainstem circuitry that controls respiratory muscles. Furthermore, comparison of these data with those obtained from previous studies in rat (Dobbins and Feldman, 1994) suggests that the organization of this circuitry is species-specific. Finally, the spatial separation of cell groups involved in the generation of the respiratory rhythm from those in the MRF that collateralize to innervate inspiratory and expiratory motoneurons suggests a functional segregation in brainstem premotor respiratory neurons that can be explored experimentally.

\section{REFERENCES}

Bartha A (1961) Experimental reduction of virulence of Aujezky's disease. Magy Allatorv Lapja 16:42-45.

Bianchi AL, Grèlot L (1989) Converse motor output of inspiratory bulbospinal premotoneurones during vomiting. Neurosci Lett 104:298-302.

Billig I, Foris JM, Card JP, Yates BJ (1999) Transneuronal tracing of neural pathways controlling an abdominal muscle, rectus abdominis, in the ferret. Brain Res 820:31-44.

Brideau AD, Banfield BW, Enquist LW (1998) The Us9 gene product of pseudorabies virus, an alphaherpesvirus, is a phosphorylated, tailanchored type II membrane protein. J Virol 72:4560-4570.

Brideau AD, Card JP, Enquist LW (2000) Role of pseudorabies virus Us9, a type IImembrane protein, in infection of tissue culture cells and the rat nervous system J Virol 74:834-845.

Card JP (1995) Pseudorabies virus replication and assembly in rodent CNS. In: Viral vectors: tools for study and genetic manipulation of the nervous system (Kaplitt MG, Loewy AD, eds), pp 319-347. San Diego: Academic.

Card JP (2000) Pseudorabies virus neuroinvasiveness: a window into the functional organization of the brain. Adv Virus Res, in press.

Card JP, Enquist LW (1999) Transneuronal circuit analysis with pseudorabies virus. In: Current protocols in neuroscience (Crawley JN, Gerfen CR, McKay R, Rogawski MA, Sibley DR, Skolnick P, eds), Unit 1.5, Supplement 9. New York: Wiley.

Dobbins EG, Feldman JL (1994) Brainstem network controlling descending drive to phrenic motoneurons in rat. J Comp Neurol 347:64-86.

Enquist LW, Card JP (1996) Pseudorabies virus: a tool for tracing neural connections and the responses of the CNS to neural infection. In: Gene transfer into postmitotic neurons: towards gene therapy of human neurological disorders (Lowenstein PR, Enquist LW, eds), pp 333-348.

Enquist LW, Husak P, Banfield BW, Smith GA (1999) Infection and spread of alphaherpesviruses in the nervous system. Adv Virus Res 51:237-347.

Feldman JL (1986) Neurophysiology of breathing in mammals. In: Handbook of physiology. The nervous system, IV. Intrinsic regulatory systems of the brain (Bloom FE, ed), pp 463-524. American Physiological Society: Bethesda, MD.

Gold H, Hatcher RA (1926) Studies on vomiting. J Pharmacol Exp Ther 28:209-218.

Grillner S, Nilsson J, Thorstensson A (1978) Intra-abdominal pressure changes during natural movements in man. Acta Physiol Scand 103:275-283. 
Hsu SM, Raine L, Fanger H (1981) Use of avidin-biotin-peroxidase complex (ABC) in immunoperoxidase techniques: a comparison between ABC and unlabeled antibody (PAP) procedures. J Histochem Cytochem 29:577-580.

Jansen ASP, Wessendorf MW, Loewy AD (1995) Transneuronal labeling of CNS neuropeptide and monoamine neurons after pseudorabies virus injections into the stellate ganglion. Brain Res 683:1-24.

Kiernan JA (1990) Histological and histochemical methods: theory and practice, Ed 2. New York: Pergamon.

Kim JS, Enquist LW, Card JP (1999) Circuit-specific coinfection of neurons in the rat central nervous system with two pseudorabies virus recombinants. J Virol 73:9521-9531.

Levatte MA, Mabon PJ, Weaver LC, Dekaban GA (1998) Simultaneous identification of two populations of sympathetic preganglionic neurons using recombinant herpes simplex virus type 1 expressing different reporter genes. Neuroscience 82:1253-1267.

Loewy AD (1990) Central autonomic pathways. In: Central regulation of autonomic functions (Loewy AD, Spyer KM, eds), pp 88-103. New York: Oxford UP.

Mabon PJ, Weaver LC, Dekaban GA (1999) Cyclosporin A reduces the inflammatory response to a multi-mutant herpes simplex virus type-1 leading to improved transgene expression in sympathetic preganglionic neurons in hamsters. J Neurovirol 5:268-279.

McCarthy LE, Borison HL (1974) Respiratory mechanics of vomiting in decerebrate cats. Am J Physiol 226:738-743.

McLean IW, Nakane PK (1974) Periodate-lysine-paraformaldehyde for immunoelectron microscopy. J Histochem Cytochem 22:1077-1083.

Miller AD, Nonaka S, Lakos SF, Tan K (1990) Diaphragmatic and external intercostal muscle control during vomiting: behavior of inspiratory bulbospinal neurons. J Neurophysiol 63:31-36.

Miller AD, Nonaka S, Jakus J, Yates BJ (1996) Modulation of vomiting by the medullary midline. Brain Res 737:51-58.
Rossiter CD, Hayden NL, Stocker SD, Yates BJ (1996) Changes in outflow to respiratory pump muscles produced by natural vestibular stimulation. J Neurophysiol 76:3274-3284.

Shiba K, Siniaia MS, Miller AD (1996) Role of ventral respiratory group bulbospinal expiratory neurons in vestibular-respiratory reflexes. J Neurophysiol 76:2271-2279.

Standish A, Enquist LW, Miselis RR, Schwaber JS (1995) Dendritic morphology of cardiac related medullary neurons defined by circuit-specific infection by a recombinant pseudorabies virus expressing betagalactosidase. J Neurovirol 1:359-368.

Ueyama T, Krout KE, Nguyen XV, Karpitskiy V, Kollert A, Mettenleiter TC, Loewy AD (1999) Suprachiasmatic nucleus: a central autonomic clock. Nat Neurosci 2:1051-1053.

Watson Jr RE, Wiegand SJ, Clough RW, Hoffman GE (1986) Use of cryoprotectant to maintain long-term peptide immunoreactivity and tissue morphology. Peptides 7:155-159.

Woodring SF, Yates BJ (1997) Responses of ventral respiratory group neurons of the cat to natural vestibular stimulation. Am J Physiol 273:R1946-R1956.

Yates BJ, Jakus J, Miller AD (1993) Vestibular effects on respiratory outflow in the decerebrate cat. Brain Res 629:209-217.

Yates BJ, Grèlot L, Kerman IA, Balaban CD, Jakus J, Miller AD (1994) Organization of vestibular inputs to nucleus tractus solitarius and adjacent structures in cat brain stem. Am J Physiol 267:R974-R983.

Yates BJ, Siniaia MS, Miller AD (1995) Descending pathways necessary for vestibular influences on sympathetic and inspiratory outflow. Am J Physiol 268:R1381-R1385.

Yates BJ, Smail JA, Stocker SD, Card JP (1999) Transneuronal tracing of neural pathways controlling activity of diaphragm motoneurons in the ferret. Neuroscience 90:1501-1513. 